\title{
Glutamate, D-(-)-2-Amino-5-Phosphonopentanoic Acid, and N-Methyl-D-Aspartate Do Not Directly Modulate Glycine Receptors
}

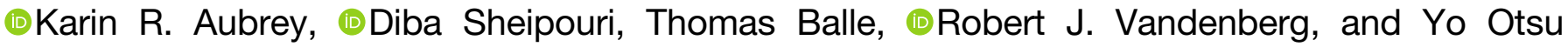

Pain Management Research Institute (K.R.A., Y.O.), Kolling Institute of Medical Research, Royal North Shore Hospital and Northern Clinical School, Faculty of Medicine and Health (K.R.A., Y.O.), Pharmacology, Faculty of Medicine and Health (D.S., R.J.V.), Sydney Pharmacy School, Faculty of Medicine and Health (T.B.), and Brain and Mind Centre (T.B.), The University of Sydney, Sydney, Australia

Received July 21, 2020; accepted September 3, 2020

\section{ABSTRACT}

Replication studies play an essential role in corroborating research findings and ensuring that subsequent experimental works are interpreted correctly. A previously published paper indicated that the neurotransmitter glutamate, along with the compounds $\mathrm{N}$-methyl-D-aspartate (NMDA) and D-(-)-2-amino5-phosphonopentanoic acid (AP5), acts as positive allosteric modulators of inhibitory glycine receptors. The paper further suggested that this form of modulation would play a role in setting the spinal inhibitory tone and influencing sensory signaling, as spillover of glutamate onto nearby glycinergic synapses would permit rapid crosstalk between excitatory and inhibitory synapses. Here, we attempted to replicate this finding in primary cultured spinal cord neurons, spinal cord slice, and Xenopus laevis oocytes expressing recombinant human glycine receptors. Despite extensive efforts, we were unable to reproduce the finding that glutamate, AP5, and NMDA positively modulate glycine receptor currents. We paid careful attention to critical aspects of the original study design and took into account receptor saturation and protocol deviations such as animal species. Finally, we explored possible explanations for the experimental discrepancy. We found that solution contamination with a high-affinity modulator such as zinc is most likely to account for the error, and we suggest methods for preventing this kind of misinterpretation in future studies aimed at characterizing high-affinity modulators of the glycine receptor.

\section{SIGNIFICANCE STATEMENT}

A previous study indicates that glutamate spillover onto inhibitory synapses can directly interact with glycine receptors to enhance inhibitory signalling. This finding has important implications for baseline spinal transmission and may play a role when chronic pain develops. However, we failed to replicate the results and did not observe glutamate, D-(-)-2-amino-5-phosphonopentanoic acid, or $\mathrm{N}$-methyl-D-aspartate modulation of native or recombinant glycine receptors. We ruled out various sources for the discrepancy and found that the most likely cause is solution contamination.

\section{Introduction}

Adult spinal cord and brainstem neurons are inhibited by synaptically released glycine and/or GABA acting at postsynaptic glycine or $\mathrm{GABA}_{\mathrm{A}}$ receptors (Kotak et al., 1998; Harvey et al., 2004; Heinke et al., 2004; Mitchell et al., 2007; Anderson et al., 2009; Yevenes and Zeilhofer, 2011; Zeilhofer et al., 2012; Foster et al., 2015, but see Moore and Trussell,

A version of this article has been submitted to the preprint server bioRxiv (https://www.biorxiv.org/).

K.R.A and Y.O are supported by the Pain Foundation Ltd. [www. painfoundation.org.au, ABN 87072480 123, Registered Charity CFN 21042]; R.J.V. and D.S. are supported by the NHMRC [Grant APP1144429]; D.S. holds a Postgraduate Scholarship from the University of Sydney; and T.B. is supported by the Australian Research Council [Grant LP160100560].

https://doi.org/10.1124/molpharm.120.000127.
2017) and excited by glutamate acting at postsynaptic ionotropic $\alpha$-amino-3-hydroxy-5-methyl-4-isoxazolepropionic acid (AMPA), kainite, or $N$-methyl-D-aspartate (NMDA) receptors. The balance between inhibition and excitation is essential for effective neuronal signaling, and disruption of this balance contributes to neuronal diseases such as autism and chronic pain (Marín, 2012; Colloca et al., 2017).

Each synapse generally uses a different fast neurotransmitter system; however, two or more neurotransmitters can coexist in some neurons (Vaaga et al., 2014). Additionally, under some circumstances, transmitters with opposing function can modulate each other's activity (Sagné et al., 1997; Chaudhry et al., 1998; Chéry and De Koninck, 1999; Ahmadi et al., 2003; Lu et al., 2008; Sun et al., 2014). For example, glycine is both an agonist at inhibitory glycine receptors (GlyRs) and a coagonist

ABBREVIATIONS: AMPA, $\alpha$-amino-3-hydroxy-5-methyl-4-isoxazolepropionic acid; AP5, D-(-)-2-amino-5-phosphonopentanoic acid; BAPTA, 1,2-bis(o-aminophenoxy)ethane-N,N,N',N'-tetraacetic acid; $95 \% \mathrm{Cl}, 95 \%$ confidence interval; elPSC, evoked inhibitory postsynaptic current; $\mathrm{GABA}_{A} \mathrm{R}, \mathrm{GABA}_{A}$ receptor; GlyR, glycine receptor; HEK, human embryonic kidney; IPSC, inhibitory postsynaptic current; mIPSC, miniature inhibitory postsynaptic current; MK-801, (5S,10R)-(+)-5-methyl-10,11-dihydro-5H-dibenzo[a,d]cyclohepten-5,10-imine; NMDA, N-methyl-D-aspartate; NMDAR, $N$-methyl-D-aspartate receptor; PAM, positive allosteric modulator; QX314, $N$-(2,6-dimethylphenylcarbamoylmethyl)triethylammonium-Cl; TTX, tetrodotoxin. 
with glutamate at excitatory $N$-methyl-D-aspartate receptors (NMDARs) (Johnson and Ascher, 1987). As a result, synaptically released glycine can spill over from inhibitory synapses onto nearby excitatory synapses and influence NMDARmediated signaling (Ahmadi et al., 2003). This form of crosstalk is thought to play an essential role in balancing inhibitory and excitatory signaling in the spinal cord and brainstem (Kullmann, 2000).

At some inhibitory hindbrain synapses, the glycine and GABA systems coexist both pre- and postsynaptically. They share a single vesicular transporter (Sagné et al., 1997; Chaudhry et al., 1998) and are copackaged in synaptic vesicles (Todd and Sullivan, 1990; Mitchell et al., 1993; Polgár et al., 2013) by most glycine releasing neurons in laminas I-III of the adult spinal cord. However, because $\mathrm{GABA}_{\mathrm{A}}$ receptors $\left(\mathrm{GABA}_{\mathrm{A}} \mathrm{Rs}_{\mathrm{S}}\right)$ and GlyR are usually differentially expressed at postsynaptic densities, mixed inhibitory transmission is rarely detected (Jonas et al., 1998; Keller et al., 2001; Aubrey et al., 2007). Instead, corelease is thought to primarily influence inhibitory transmission by engaging extrasynaptic and presynaptic receptors (Chéry and De Koninck, 1999, 2000; Lim et al., 2000; Keller et al., 2001; Turecek and Trussell, 2001), although GlyR decay kinetics are allosterically modulated by GABA (Lu et al., 2008).

In a paper published in the journal Nature Neuroscience in 2010, Liu and colleagues reported that glutamate allosterically facilitates GlyR-mediated currents with a maximal enhancement of $\sim 200 \%$ (Liu et al., 2010). They also showed that the amplitude of glycinergic IPSCs increased when the competitive NMDAR antagonist D-(-)-2-amino-5-phosphonopentanoic acid (AP5) and a range of other compounds that interact with the glutamate binding sites on glutamate receptors (e.g., NMDA, kainic acid, and quisqualate) were present. In contrast, the pore blocking NMDAR antagonist (5S,10R)-(+)-5-methyl-10,11-dihydro-5H-dibenzo[a,d]cyclohepten-5,10-imine (MK-801) and competitive AMPA receptor antagonists NBXQ (2,3-Dioxo-6-nitro-1,2,3,4-tetrahydrobenzo [f]quinoxaline-7-sulfonamide) and CNQX (6-Cyano-7-nitroquinoxaline-2,3-dione) had no apparent effect. The observation was verified in human embryonic kidney (HEK) cells expressing recombinant GlyRs, suggesting a direct interaction of glutamate and its analogs with GlyRs. Single-channel recordings showed that AP5 increased the open dwell time of native GlyRs without changing conductance levels and that the AP5 enhancement relied on the drug being on the extracellular side of the receptor. Finally, the paper showed that the amplitude of glycinergic miniature IPSCs recorded in rat spinal cord slices was increased by $42 \%$, without any change in frequency, when threo- $\beta$-benzyloxyaspartic acid, a glutamate transporter antagonist, was used to elevate the synaptic concentration of glutamate. These findings are significant because they suggest that the size and duration of native GlyR currents will be determined not only by the relative concentration of glycine and GABA in synapses but also by the extent of glutamate spillover from nearby synapses. Additionally, these findings suggested that all in vitro measures of GlyR currents that were carried out in the presence of AP5 or kynurenic acid should be reevaluated and indicated that these commonly used compounds should habitually be excluded from electrophysiological recordings of IPSCs.

The hypothesis that glycine and glutamate spillover reciprocally modulates NMDAR- and GlyR-mediated transmission under normal and pathologic (chronic pain) conditions and the functional outcome of altering this modulation have never been investigated. We sought to replicate the finding that AP5, NMDA, and glutamate act as positive allosteric modulators (PAMs) of GlyRs (Liu et al., 2010) by recording GlyRmediated currents from whole-cell patch clamped mouse primary cultured spinal cord neurons and from oocytes expressing recombinant GlyRs. Surprisingly, we were unable to find any evidence that AP5, NMDA, or glutamate facilitate glycine currents; instead, we observed no change or a small reduction in current amplitudes. Next, we looked for an explanation for the discrepancy between our findings and the original experiments by Liu et al. We found that neither species difference nor indirect modulation by calcium is the likely cause. However, nanomolar levels of zinc, a high-affinity GlyR PAM could cause a similar GlyR enhancement.

\section{Materials and Methods}

\section{Ethical Approval}

All procedures involving animals followed the guidelines of the "Australian Code of Practice for the Care and Use of Animals for Scientific Purposes" and had the approval of the Royal North Shore Hospital Animal Ethics Committee (rodent work) or the University of Sydney Animal Ethics Committee (Xenopus).

Rats were housed in groups of two to three and mice in groups of up to five in individually ventilated cages under a 12:12 hour light/dark cycle, with environmental enrichment and free access to water and standard rodent chow.

\section{Recordings from Neurons}

Embryonic Mouse Spinal Cord Neurons. Primary cultures of spinal cord neurons were prepared as described by Hanus (2004) (see also Rousseau et al., 2008) from embryonic day 13 to 14 C57BL/6 wildtype or GlyT2::Cre $\times$ mTmG mouse pups. Embryos were obtained by cesarean section from pregnant mice $(n=12)$ deeply anesthetized with isoflurane (3\%; assessed by rate of breathing, lack of righting reflexes, and lack of withdrawal reflex in response to hind paw squeeze) and killed by cervical dislocation. Spinal cords were dissected under sterile conditions into PBS with $33 \mathrm{mM}$ glucose at $\mathrm{pH} 7.4$ and then incubated in trypsin/EDTA solution $(0.05 \% \mathrm{v} / \mathrm{v})$ for 10 minutes at $37^{\circ} \mathrm{C}$. Cells were dissociated mechanically in a modified L15 Leibowitz's medium (Invitrogen) and plated at a density of $1.4-2.0 \times 10^{5}$ cells $/ \mathrm{cm}^{2}$ on sterilized glass coverslips coated with $60 \mu \mathrm{g} / \mathrm{ml}$ poly- $d l$-ornithine and with medium containing $5 \%$ inactivated fetal calf serum (Sigma). Cells were maintained at $37^{\circ} \mathrm{C}$ in $5 \% \mathrm{CO}_{2}$ in serum-free Neurobasal plus medium containing supplement B27 plus (Invitrogen) for up to 3 weeks. One-third of the medium was replaced every 3 to 4 days.

Spinal cord slices were obtained from two male adult Sprague Dawley rats (8-10 weeks old) that had vector-mediated channel rhodopsin expressed in rostroventral medial medulla neurons. Rats were obtained from the Animal Resources Centre (Canning Vale, Australia). Rats were deeply anesthetized with isoflurane (3\%, assessed by rate of breathing, lack of righting reflexes, and lack of response to paw squeeze), transcardially perfused with ice-cold $\mathrm{N}$-methyl-D-glucamine solution (in millimolars, $93 \mathrm{~N}$-methyl-D-glucamine; $30 \mathrm{NaHCO}_{3} ; 25$ glucose; 2 thiourea; $3 \mathrm{Na}$-pyruvate; $2.5 \mathrm{KCl} ; 1.2$ $\mathrm{NaH}_{2} \mathrm{PO}_{4}-\mathrm{H}_{2} \mathrm{O} ; 20$ HEPES; $10 \mathrm{MgSO}_{4}-7 \mathrm{H}_{2} \mathrm{O} ; 0.5 \mathrm{CaCl}_{2} ; 5$ sodium ascorbate; $300 \mathrm{mOsm}$, equilibrated with $95 \% \mathrm{O}_{2}-5 \% \mathrm{CO}_{2}$ ) and parasagittal spinal cord slices $(280 \mu \mathrm{m})$ of the lumbar enlargement were prepared with a vibratome (VT1200S; Leica Microsystems AG, Wetzlar, Germany) in the same solution. After maintaining the slices for 10 minutes at $34^{\circ} \mathrm{C}$ in a submerged chamber, they were kept in artificial cerebrospinal fluid equilibrated with $95 \% \mathrm{O}_{2}$ and $5 \% \mathrm{CO}_{2}$ until recording. The slices were then individually transferred to 


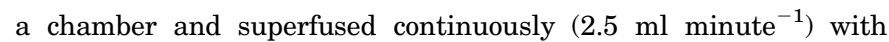
artificial cerebrospinal fluid $\left(32^{\circ} \mathrm{C}\right.$, composition, (in millimolars: 126 $\mathrm{NaCl}, 2.5 \mathrm{KCl}, 1.4 \mathrm{NaH}_{2} \mathrm{PO}_{4}, 1.2 \mathrm{MgCl}_{2}, 2.4 \mathrm{CaCl}_{2}, 11$ glucose, and 25 $\mathrm{NaHCO}_{3}$ ).

Electrophysiology. Whole-cell voltage-clamp recordings of cultured spinal cord neurons (12-22 days in vitro) or lamina II neurons in rat parasagittal spinal cord slice were performed at $\sim 32^{\circ} \mathrm{C}$ using a Multiclamp 700B controlled and Axograph acquisition software. Currents were filtered at $4 \mathrm{kHz}$ and sampled at $20 \mathrm{kHz}$ using a National Instruments USB-6251 digitizer for online and later offline analysis (Axograph 1.7.6). A sample size of four to eight cells per group was anticipated prior to the experiments based on previous experience and the results of Liu et al. (2010). Cells where the series resistance of the recorded neuron was greater than $25 \mathrm{M} \Omega$, and where it changed by more than $25 \%$, were excluded from the analysis. Patch pipettes were pulled from borosilicate glass capillaries (Hilgenberg) and had resistances of 4-6 M $\Omega$. Miniature and evoked currents were recorded at a holding potential of -60 or $-65 \mathrm{mV}$ using pipettes filled with internal solution containing the following (in millimolars): $140 \mathrm{CsCl}$, $1 \mathrm{CaCl}_{2}, 1 \mathrm{MgCl}_{2}, 10$ EGTA, 1 1,2-bis(2-aminophenoxy)ethane$\mathrm{N}, \mathrm{N}, \mathrm{N}^{\prime}, \mathrm{N}^{\prime}$-tetraacetic acid (BAPTA), $4 \mathrm{Mg}$-ATP, 5 QX314 [N-(2,6dimethylphenylcarbamoylmethyl)triethylammonium-Cl], and 10 HEPES (pH 7.4) with $\mathrm{CsOH}, 300 \pm 5$ mOsm. In Fig. 4 the internal solutions were modified; $10 \mathrm{mM}$ BAPTA intracellular solution contained (in millimolars) $140 \mathrm{CsCl}, 10 \mathrm{BAPTA}, 4 \mathrm{Mg}$-ATP and 5 QX314 and 10 HEPES (pH 7.20, osmolality, $295 \pm 5$ mOsm) (Liu et al., 2010), and $0.6 \mathrm{mM}$ EGTA intracellular solution contained (in millimolars): 140 CsCl, $1 \mathrm{MgCl}_{2}$, 0.6 EGTA, 4 Mg-ATP, 5 QX314 [ $N$-(2,6-dimethylphenylcarbamoylmethyl)triethylammonium-chloride], and $10 \mathrm{HEPES} \mathrm{pH}$ 7.4 with $\mathrm{CsOH}, 300 \pm 5 \mathrm{mOsm}$ ). In Fig. $4 \mathrm{~B}$, neurons were exposed to NMDA for $>5$ minutes before miniature IPSC (mIPSC) were analyzed (Kloc et al., 2019).

For paired recordings, the internal solution for the presynaptic current clamped neuron contained (in millimolars): 155 potassiumgluconate, $4 \mathrm{KCl}, 5 \mathrm{Mg}$-ATP, $0.1 \mathrm{EGTA}$, and $10 \mathrm{HEPES}$, adjusted to $\mathrm{pH}$ 7.4 with $\mathrm{KOH}$. Neurons were continuously bathed with an external solution containing the following (millimolars): $140 \mathrm{NaCl}, 5.4 \mathrm{KCl}, 10$ HEPES, $1 \mathrm{MgCl}_{2}, 1.3 \mathrm{CaCl}_{2}$ and 20 glucose ( $\mathrm{pH} 7.4$, 305-315 mOsm). AMPA and NMDA receptors were blocked with $10 \mu \mathrm{M}$ CNQX and when indicated $15 \mu \mathrm{M}$ MK-801. GABA $\mathrm{A}_{\mathrm{A}}$ were selectively blocked with $10 \mu \mathrm{M}$ bicuculline. When indicated, GlyRs were blocked with $0.5-1 \mu \mathrm{M}$ strychnine. Miniature events were recorded in the presence of $0.5 \mu \mathrm{M}$ tetrodotoxin (TTX). A concentration of AP5 equivalent to $100 \mu \mathrm{M} d$-AP5 was used in all experiments unless otherwise indicated (e.g., $100 \mu \mathrm{M} d$-AP5 or $200 \mu \mathrm{M} d l$-AP5), NMDA was used at $50 \mu \mathrm{M}$, and glutamate at $100 \mu \mathrm{M}$. Drugs were purchased from Sigma Aldrich or Tocris.

\section{Two-Electrode Voltage-Clamp Recording of Heterologously Expressed Human Glycine Receptors in Xenopus laevis Oocytes}

Human GlyR $\alpha 1, \alpha 3, \alpha 1 \beta$, and $\alpha 3 \beta$ cDNA were subcloned into pGEMHE. The amplified cDNA/pGEMHE product was then transformed in Escherichia coli cells and subsequently purified using the PureLink Quick Plasmid Miniprep Kit (Invitrogen by Life Technologies, Löhne, Germany) and sequenced by the Australian Genome Research Facility (Sydney, Australia). The purified plasmid DNA was linearized using the restriction enzyme NheI [New England Biolabs (Genesearch), Arundel, Australia]. Complementary RNAs were synthesized using the mMESAGE mMACHINE T7 kit (Ambion, TX).

Xenopus laevis frogs $(n=4)$ were anesthetized with $0.17 \%(\mathrm{w} / \mathrm{v}) 3-$ aminobenzoic acid ethyl ester, and surgical anesthesia was assessed by a regular and relaxed respiratory rate, no withdrawal reflex when the hind feet were pinched, no muscle tone when the hind limb was extended, and no response to external stimuli. Then, an ovarian lobe was removed via an incision in the abdomen and stage $\mathrm{V}$ oocytes were isolated from the lobe via digestion with $2 \mathrm{mg} / \mathrm{ml}$ collagenase A
(Boehringer, Mannheim, Germany) at $26^{\circ} \mathrm{C}$ for 1 hour. Two nanograms of cRNA encoding GlyR $\alpha 1$ or $\alpha 3$ was injected into each oocyte cytoplasm when the receptors were studied individually. Where GlyR $\alpha 1 \beta$ or $\alpha 3 \beta$ was expressed, a 1:5 ratio of GlyR $\alpha 1 / 3$ (2 ng) and GlyR $\beta$ (10 ng) cRNA was injected into single cells, as this ratio was found to be sufficient for the formation of GlyR heteromers as judged by reduced sensitivity to picrotoxin compared with GlyR $\alpha 1$ or $\alpha 3$ homomers. The oocytes were then stored in frog Ringer's solution (in millimolars: $96 \mathrm{NaCl}, 2 \mathrm{KCl}, 1 \mathrm{MgCl}_{2}, 1.8 \mathrm{CaCl}_{2}, 5 \mathrm{mM}$ HEPES, $\mathrm{pH}$ 7.5), which was supplemented with $2.5 \mathrm{mM}$ sodium pyruvate, $0.5 \mathrm{mM}$ theophylline, $50 \mu \mathrm{g} / \mathrm{ml}$ gentamicin, and $100 \mu \mathrm{M} \mathrm{ml}^{-1}$ tetracycline. The oocytes were stored at $18^{\circ} \mathrm{C}$ for $2-5$ days until receptor expression was adequate for measurement using the two-electrode voltage-clamp technique. Oocytes were voltage-clamped at $-60 \mathrm{mV}$, and whole-cell currents generated by the substrate were recorded with a Geneclamp 500 amplifier (Axon Instruments, Foster City, CA) and a Powerlab 2/20 chart recorder (ADInstruments, Sydney, Australia).

\section{Data Analysis}

A sample size of four to eight cells per group was anticipated prior to the experiments based on previous experience and the results of Liu et al. (2010). $P$ values were assessed using two-tailed paired $t$ tests and one-way ANOVAs.

mIPSCs were detected using a template protocol $(\mathrm{SD}=-3.5)$ in Axograph. mIPSCs that had multiple peaks, were less than $10 \mathrm{pA}$ in amplitude or had rise time and decay time values outside $0.1-1$ and 1-10 milliseconds, respectively, were discarded. mIPSCs were detected over 1-5 minutes before and after drug exposure. mIPSC frequency as calculated in $\mathrm{Hz}$, and the peak amplitude and charge transfer of mIPSC currents were measured from the average mIPSC current. Charge transfer was measured from the mIPSC area (picoampere.millisecond) and calculated from the beginning of the mIPSC onset until the current returned to baseline. Charge transfer reflects the combined peak amplitude and kinetic of mIPSCs.

In Figure 5, the effect of the GlyR PAM zinc was investigated. Zinc was extracellularly applied at a concentration of $50 \mathrm{nM}$ or $2 \mu \mathrm{M}$ free zinc (5 or $200 \mu \mathrm{M}$ zinc chloride in buffer containing the zinc chelator tricine, $10 \mathrm{mM}$; Paoletti et al., 1997).

The peak current, charge, and frequency of mIPSCs were compared with two-tailed paired $t$ tests, and the mean value and $95 \%$ confidence interval (95\% CI) of the normalized values (drug-treated/control) were reported.

Evoked IPSC peak currents were measured before and after exposure to AP5 by averaging five consecutive evoked IPSC (eIPSC) currents (triggered every 30 seconds). eIPSCs were measured after $>1$ minute preincubation with drugs. Three out of five eIPSC experiments presented included the NMDA receptor antagonist MK-801 $(15 \mu \mathrm{M})$ in the bath solution, but as the results were not altered by the inclusion of this drug, the data were pooled. The peak current and kinetics of eIPSCs were compared with a two-tailed paired $t$ test, and the mean and $95 \%$ confidence interval of the normalized amplitude (AP5/control) value was reported.

Exogenous glycine currents were induced by extracellular $10-50 \mu \mathrm{M}$ glycine applied for 1-3 seconds \pm drug with a fast-step system. Glycine was applied every 1 minute to allow glycine to wash out from the bath and to minimize the effects of receptor desensitization. Current amplitudes were measured by averaging three consecutive currents before and after exposure to drugs (AP5, NMDA). The current amplitudes were compared with a two-tailed paired $t$ test, and the mean and 95\% confidence interval of the normalized (drug-treated/ control) values were reported. In Fig. 2, when NMDA was applied, a modified extracellular solution was used to prevent NMDARmediated effects. This extracellular solution was $\mathrm{Ca}^{2+}$ free and contained EDTA (10 mM) and $15 \mu \mathrm{M}$ MK-801.

Oocytes expressing GlyRs were placed in an oval-shaped bath with a volume of $0.5 \mathrm{ml}$, with laminar flow of frog Ringer's solution around the oocyte at a rate of $10 \mathrm{ml}$ minute $^{-1}$ under gravity feed. Glutamate, NMDA, and $d$-AP5 were bath applied to oocytes for $\sim 1$ minute before 
coapplication with glycine and measurement of whole-cell glycinestimulated peak currents. Consecutive currents were stimulated 5 minutes apart to allow glycine to washout of the bath and to minimize the effects of receptor desensitization. The mean and 95\% confidence interval of the normalized amplitude of currents (drug-treated/control) were reported.

Picrotoxin Assay. An approximate $\mathrm{EC}_{70}$ concentration of glycine, or concentration as indicated, was applied to oocytes until the current amplitude reached a plateau. Increasing concentrations of picrotoxin were then coapplied with glycine until the change in response plateaued. Current values at those plateaus were measured and compared with the initial response to glycine in the absence of picrotoxin. Dose responses were fitted to the equation

$$
\left.\mathrm{Y}=\text { Bottom }+(\text { Top-Bottom }) /\left(1+10\left(\log _{50}-\mathrm{X}\right) * \text { HillSlope }\right)\right)
$$

where $\mathrm{X}$ is log [picrotoxin] (micromolars), $\mathrm{Y}$ is current normalized to the response generated by glycine in the absence of picrotoxin, the Top and Bottom are plateaus at the maximum and minimum values for log [picrotoxin], and the HillSlope is the steepness of the curve.

\section{Results}

AP5 Does Not Enhance Synaptic Glycine Currents. We recorded GlyR mIPSCs from cultured mouse spinal cord neurons in the presence and absence of the competitive NMDAR antagonist AP5. AP5 did not substantially alter glycine mIPSC peak amplitude, charge transfer, or frequency (Fig. 1, A-F) compared with control conditions [normalized amplitude $(\mathrm{AP} 5 /$ control $)=0.98 ; 95 \%$ confidence interval: $0.74-1.15, P=0.88$; normalized charge $(\mathrm{AP} 5 /$ control $)=0.98$; 95\% confidence interval: $0.82-1.23, P=0.96$; normalized frequency $(\mathrm{AP} 5 /$ control $)=1.05 ; 95 \%$ confidence interval: $0.83-1.28, P=0.64$, paired two-tailed $t$ test]. Likewise, when we recorded glycinergic eIPSCs from pairs of connected neurons, AP5 did not substantially alter the glycine eIPSC peak amplitude or kinetics [Fig. 1, G-I; normalized amplitude $(\mathrm{AP} 5 /$ control $)=0.92 ; 95 \%$ confidence interval: $0.75-1.08$, $P=0.33]$.

AP5 and NMDA Do Not Enhance Extrasynaptic Receptors. In contrast to eIPSCs and mIPSCs, short application of
A
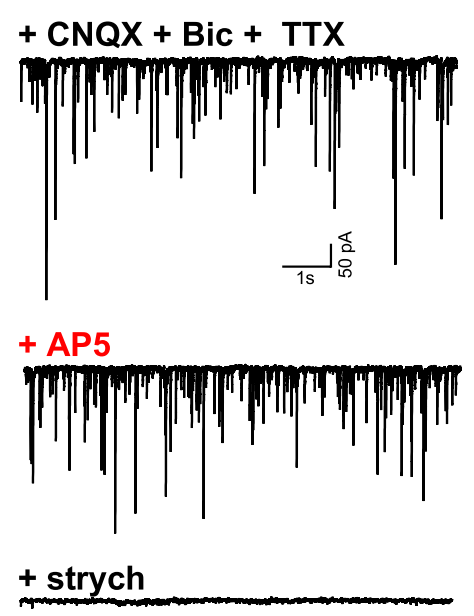

B

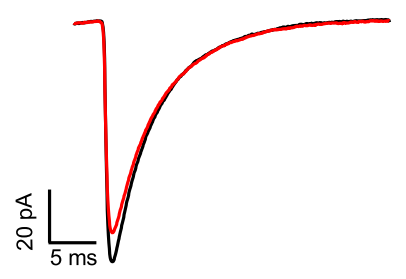

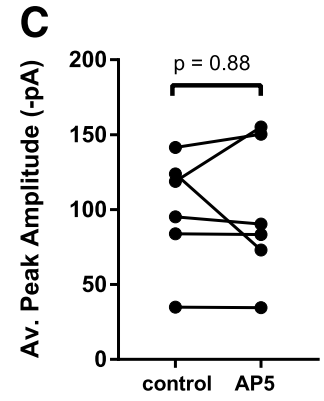

D
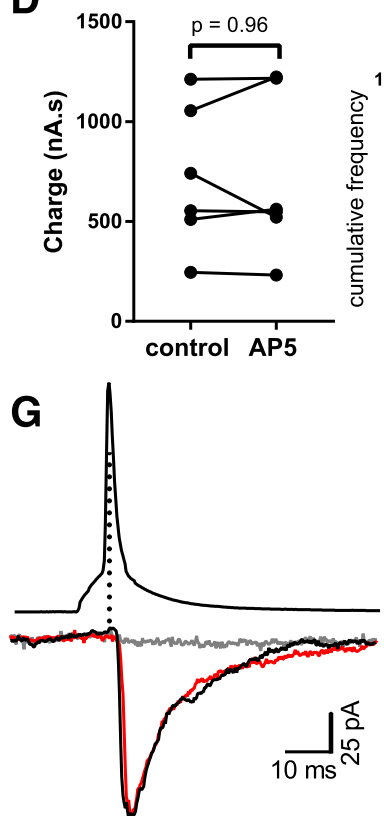

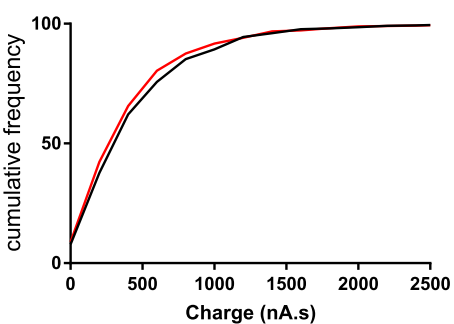

H

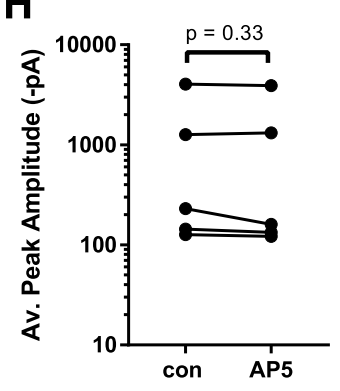

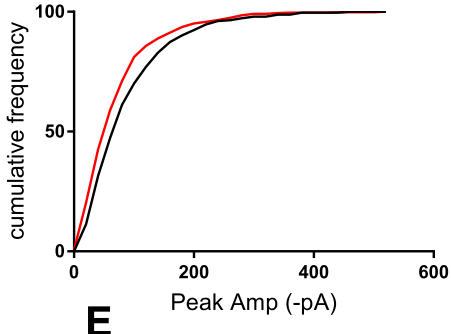

E

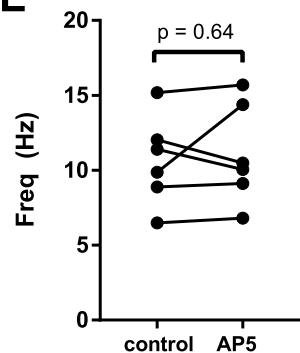

I

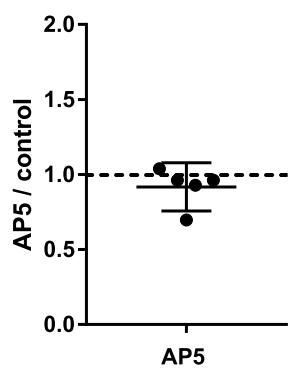

Fig. 1. Glycine mIPSC and eIPSCs recorded in cultured spinal cord neurons are not altered by AP5. (A) Example traces of mIPSCs recorded in control conditions and after the addition of AP5 and then strychnine (strych) to the bath solution. (B) mIPSC were compared before (black line) and after (red line) exposure to $100 \mu \mathrm{M}$ AP5. (C-E) mIPSC peak amplitude (C), charge transfer (D), and frequency (E) were measured from individual cells. Cumulative frequency plots are shown for peak amplitude (C) and charge (D). Paired data from each experiment are connected by a line (C-E). (F) AP5 did not alter mIPSC amplitude, charge transfer, or frequency relative to their control values (AP5/control). (G) A depolarizing current-step triggers an action potential in a current-clamped presynaptic neuron that evokes an eIPSC in a connected voltage-clamped postsynaptic neuron held at a holding potential of $-65 \mathrm{mV}$. The eIPSC (black line) amplitude and kinetic was not altered by exposure to $100 \mu \mathrm{M}$ AP5 (red line). (H, I) The average peak amplitude of eIPSCs from individual pairs before and after AP5 application. Each individual experiment is connected by a line (H), and the proportional change (AP5/control) from individual experiments is shown in (I). A straight line marks the mean change, and error bars show its $95 \%$ confidence interval. con, control. Bic, Bicuculline; CNQX, 6-Cyano-7-nitroquinoxaline-2,3-dione. 
extracellular glycine activates both extrasynaptic and synaptic receptors. Extrasynaptic receptors are likely to be constructed from a more diverse range of GlyR subunits and interact with distinct partner proteins (Triller and Choquet, 2005). To test the hypothesis that AP5 modulates extrasynaptic forms of GlyRs, we recorded extracellular glycine evoked currents $(50 \mu \mathrm{M})$ in the presence and absence of AP5. Glycine induced currents were not enhanced by AP5 (Fig. 2, A, B, and D) [(50 G + AP5 current)/(50 G current $)=0.93,95 \%$ confidence interval: $0.88-0.98, P=0.07]$. When we reduced the saturation of GlyRs by lowering the glycine concentration to $10-30 \mu \mathrm{M}$ glycine (Fig. 2, C and D), no AP5 effect was observed (10-30 $\mu \mathrm{M}$ glycine + AP5, 0.93 of control; 95\% confidence interval: $0.83-1.03 ; P=0.24)$. As the selective NMDAR agonist NMDA was also reported to be an effective GlyR activator (Liu et al., 2010), we assessed its ability to alter glycine currents when applied alone or when coapplied with AP5. Once again, no effect was observed (Fig. 2, E-G) [(10 G + NMDA current $) /(10 \mathrm{G}$ current $)=0.943$, confidence interval: 0.75-1.14; $(10 \mathrm{G}+\mathrm{NMDA}+\mathrm{AP} 5$ current $) /(10 \mathrm{G}$ current $)=0.85$ of control; confidence interval: 0.58-1.13; adjusted $P=0.16$, repeat measure one-way ANOVA]. Recordings with NMDA were carried out in a modified extracellular solution that was $\mathrm{Ca}^{2+}$ free and contained EGTA and MK-801 to prevent NMDAR-mediated effects.

Glutamate Does Not Alter Recombinant GlyR Currents. In the original study, recombinant $\alpha 1$ and $\alpha 1 \beta$ GlyR currents expressed in HEK cells were reported to be enhanced by glutamate, AP5, and NMDA, as well as a host of other glutamate-like ligands (Liu et al., 2010). This finding is important for two reasons. Firstly, mature GlyRs are pentameric channel complexes made from a combination of $\alpha 1, \alpha 3$, and $\beta$ subunits. $\alpha 1 \beta$-containing GlyRs are the most common form, and $\alpha 3$-containing GlyRs are involved in inhibitory signaling in the spinal cord dorsal horn (Harvey et al., 2004). Secondly, HEK cells do not express the multitude of neuronal receptors, signaling molecules, and receptor-associated binding proteins found in neurons. Thus, AP5 facilitation of HEK-expressed recombinant GlyRs strongly suggests the presence of a unique PAM binding site for these compounds on mature forms of GlyRs.

To test if we could replicate this finding, we assessed the ability of glutamate, NMDA, and AP5 to alter whole-cell glycine currents in Xenopus oocytes expressing recombinant human $\alpha 1$ and $\alpha 1 \beta$ glycine receptors. We extended this work to include oocytes expressing recombinant $\alpha 3$ and $\alpha 3 \beta$ GlyRs (Fig. 3) as $\alpha 3$-containing GlyRs would have been engaged in the slice experiments presented in Liu et al. (2010) and cultured embryonic spinal cord neurons do not express measurable levels of $\alpha 3$-containing GlyRs [prostaglandin E2 (Harvey et al., 2004) failed to inhibit glycinergic mIPSCs in our cultures; normalized amplitude (PGE2/control) $=1.07$, 95\% confidence interval: $0.96-1.17, P=0.29$; normalized frequency $(\mathrm{PGE} 2 / \mathrm{control})=1.04,95 \%$ confidence interval: $0.73-1.35 ; P=0.69, n=7$, two-tailed paired $t$ test, data not shown].

Glycine (5-20 $\mu \mathrm{M})$ induced a large inward current that was entirely inhibited by strychnine (data not shown). Glutamate (Fig. 3, A-E), AP5, and NMDA (Fig. 3, F and G, 100, 50, $50 \mu \mathrm{M}$, respectively) did not stimulate any current when applied alone, and the mean amplitude of glycine-induced currents in all GlyR subtypes tested was not altered by their presence
A

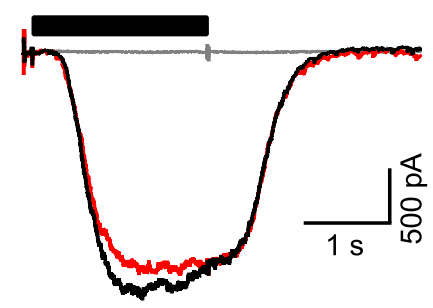

E

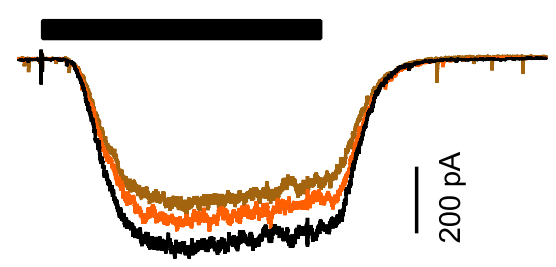

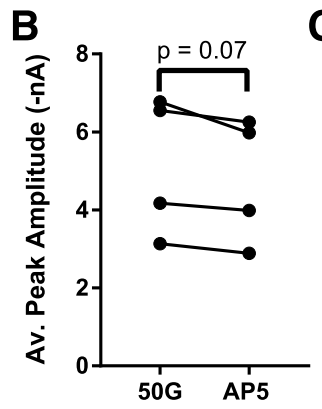
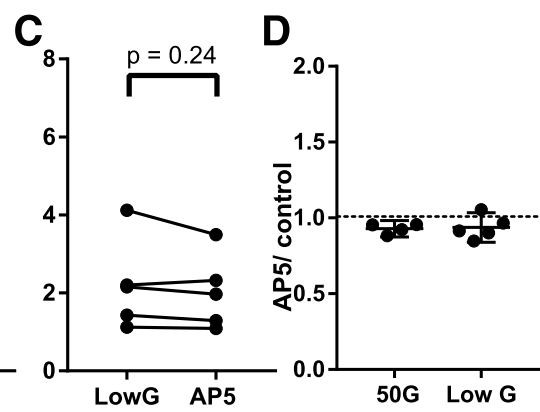

F

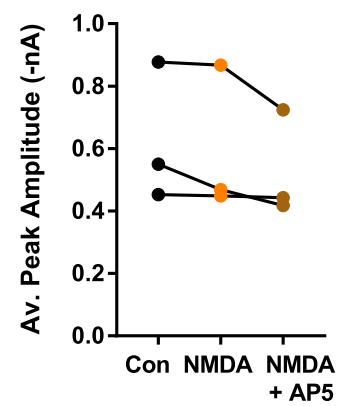

G

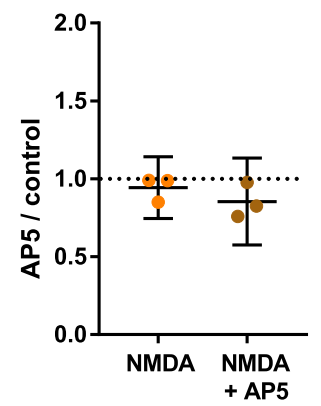

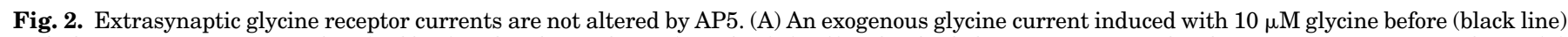

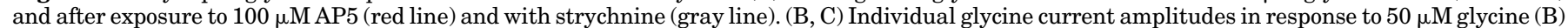

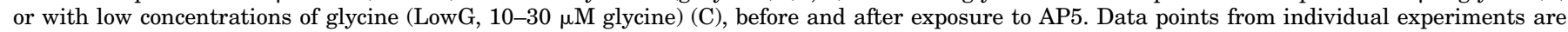

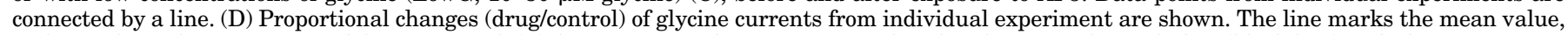

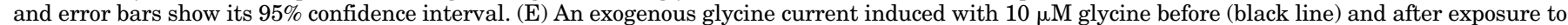

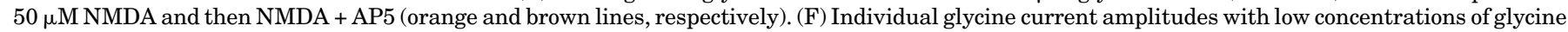

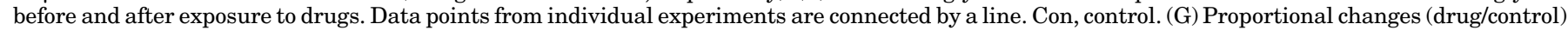
of glycine currents from individual experiment are shown. The line marks the mean value, and error bars show its $95 \%$ confidence interval. 
[GlyR $\alpha 1$ normalized current amplitude $=0.99,95 \%$ confidence interval: $0.83-1.14$ (glutamate), $=0.88,95 \%$ confidence interval: $0.62-1.14$ (AP5), = 0.97, 95\% confidence interval: 0.73-1.21 (NMDA), $P=1.00$; GlyR $\alpha 1 \beta$ normalized current amplitude $=0.78,95 \%$ confidence interval: $0.65-0.91$ (glutamate), =0.81, 95\% confidence interval: 0.64-0.97 (AP5), = 1.07, 95\% confidence interval: $0.85-1.29$ (NMDA), $P=0.23$; GlyR $\alpha 3=0.90,95 \%$ confidence interval: $0.75-1.05$ (glutamate), =0.97, 95\% confidence interval: 0.88-1.06 (AP5), = 0.99 , 95\% confidence interval: $0.84-1.14$ (NMDA), $P=0.96$;
GlyR $\alpha 3 \beta$ normalized current amplitude $=0.87,95 \%$ confidence interval: $0.78-0.97$ (glutamate), $=0.86,95 \%$ confidence interval: $0.71-1.01$ (AP5), = 0.95, 95\% confidence interval: 0.81-1.10 (NMDA), $P=0.83$, one-way ANOVA and Tukey's multiple comparisons test].

Example traces for each GlyR subunit composition with glutamate coapplication are shown in Fig. 3, A-D, and Fig. 3, E-G summarizes the data for AP5 and NMDA coapplications. The inserts (Fig. 3i and ii) show the differential effect of picrotoxin on $\alpha$-homomeric and $\alpha \beta$ heteromeric GlyRs.

A
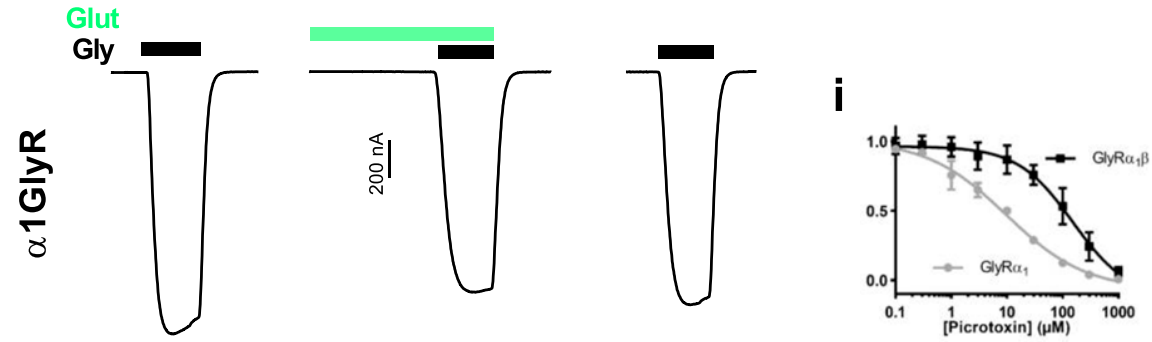

B
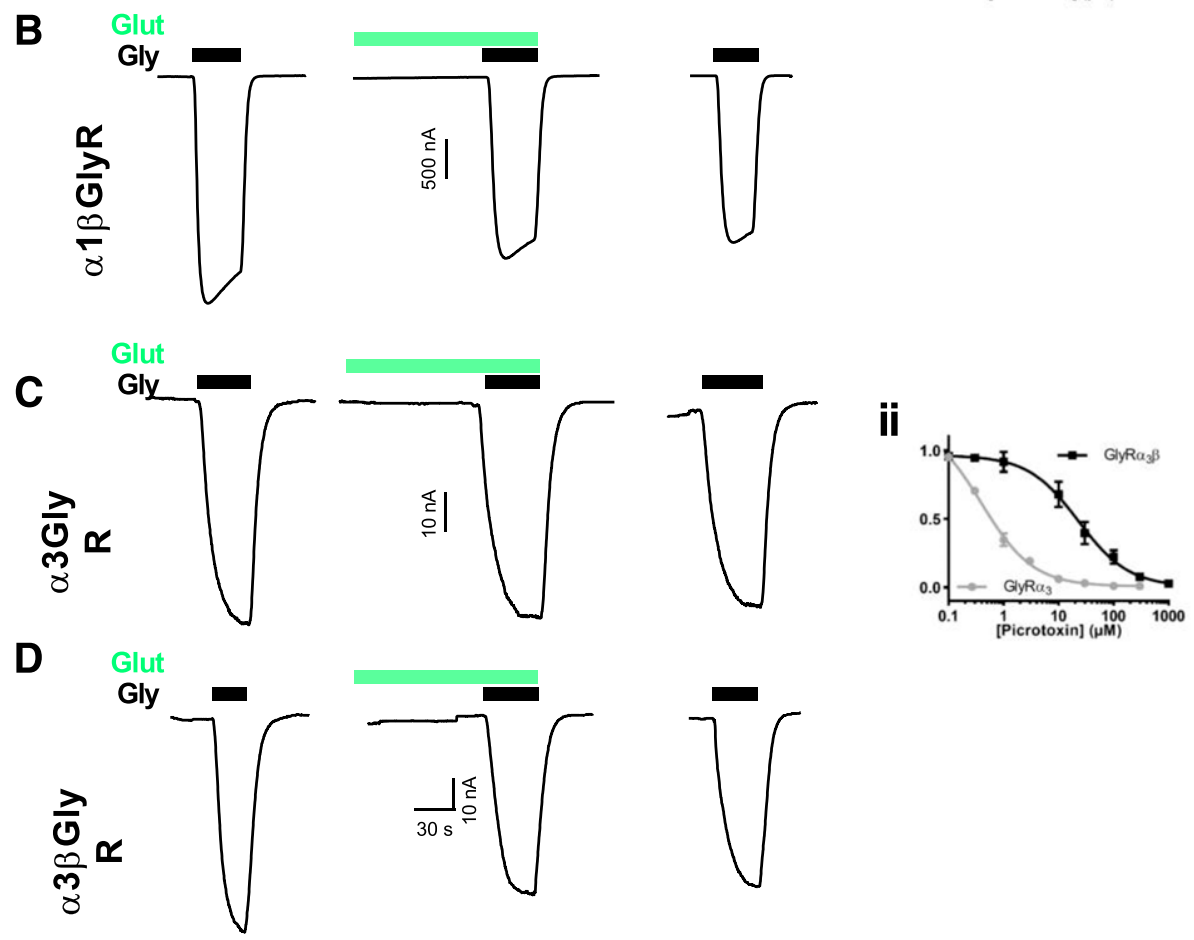

E

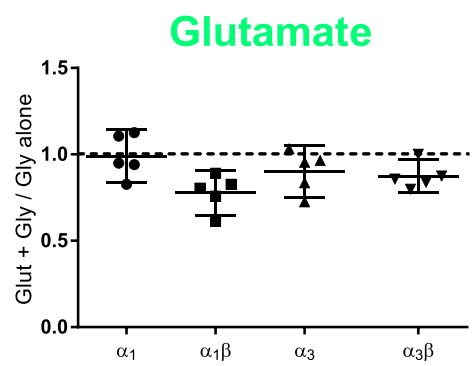

$\mathbf{F}$

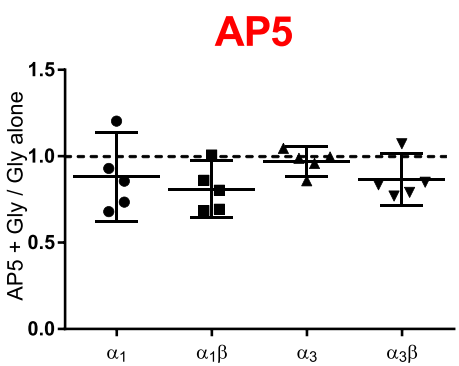

G

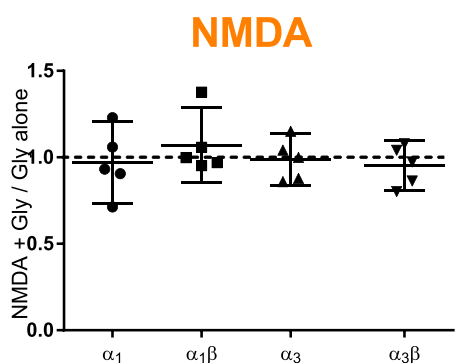

Fig. 3. (A-D) Glycine evoked currents recorded in oocytes expressing recombinant $\alpha 1$ (A), $\alpha 1 \beta$ (B), $\alpha 3$ (C), or $\alpha 3 \beta$ (D) glycine receptors were not modulated by glutamate, AP5, or NMDA. Examples of currents induced by extracellular application of glycine (5-10 $\mu \mathrm{M}$, black bar) before and after glutamate preincubation ( $100 \mu \mathrm{M}$, gray bar). Inserted graphs (i and ii) show the sensitivity of $\alpha 1 \beta$ - (top) and $\alpha 3 \beta$ - (bottom) GlyR receptor subtypes to picrotoxin to confirm the expression of the desired receptor compositions. (E-G) Proportional changes (drug/control) of glycine currents from individual experiments are shown in the presence of glutamate (E), AP5 (F), or NMDA $(\mathrm{G})$. The line marks the mean value, and error bars show its $95 \%$ confidence interval from $n=5$ oocytes in each condition. 
The 10-fold decrease in picrotoxin sensitivity when $\beta$ subunits are present indicates that $\alpha 1 \beta$ or $\alpha 3 \beta$ heteromers have been formed.

As we were unable to replicate the finding that glutamate, AP5, and NMDA allosterically potentiate glycine receptors in native or recombinant systems, we considered the possible sources for this discrepancy and carried out a series of experiments to test the validity of our theories.

Calcium Buffering Was Sufficient. Transient elevations in intracellular calcium levels are known to enhance the amplitude of GlyR currents by causing an increase in GlyR single-channel open probability (Fucile et al., 2000) and increasing GlyR retention in postsynaptic active zone clusters (Lévi et al., 2008). This form of potentiation is indirect (in contrast to the effect reported by Liu et al., 2010), as it is dependent on calcium/calmodulin-dependent protein kinase II (Xu et al., 2000; Yamanaka et al., 2013) and is prevented by including calcium chelators such as EGTA and BAPTA in the intracellular solutions (Kloc et al., 2019; Xu et al., 2000). The $\mathrm{Ca}^{2+}$-dependent GlyR current enhancement occurs over time frames compatible with the results reported by Liu et al. (2010) and can be stimulated by NMDA and NMDAR activation (Fucile et al., 2000; Xu et al., 2000; Lévi et al., 2008; Kloc et al., 2019). Our intracellular solution contained $1 \mathrm{mM}$ BAPTA + $10 \mathrm{mM}$ EGTA + $1 \mathrm{mM} \mathrm{Ca}^{2+}$, which strongly limits intracellular calcium transients. The original study used intracellular solution that contained $10 \mathrm{mM}$ BAPTA $+0 \mathrm{mM}$ $\mathrm{Ca}^{2+}$. In both cases, the extracellular solution contained $1.3 \mathrm{mM} \mathrm{Ca}{ }^{2+}$. We exactly replicated the intracellular and extracellular solutions of Liu et al. (2010) and recorded mIPSCs in the presence and absence of AP5 to confirm that $10 \mathrm{mM}$ BAPTA dissolved in the intracellular solution and no AP5 effect was observed [normalized amplitude (AP5/control) $=$ 1.1, 95\% confidence interval: $0.98-1.25, P=0.19$; normalized charge $($ AP5/control $)=1.1,95 \%$ confidence interval: $0.90-1.33$, $P=0.24$; normalized frequency $(\mathrm{AP} 5 /$ control $)=1.31,95 \%$ confidence interval: $0.74-1.88, P=0.33$, two-tailed paired $t$ tests].

To test the theory that an enhancement of GlyR mIPSCs could be observed under our recording conditions when the recorded neuron had a low intracellular buffering capacity, we recorded mIPSCs using an intracellular solution that contained $0.6 \mathrm{mM}$ EGTA + $0 \mathrm{mM} \mathrm{Ca}^{2+}$ (Fig. 4B). NMDARdependent increases in glycine eIPSCs have been observed in mouse spinal cord slices when recorded with 0.6 mM EGTAcontaining intracellular solution (Kloc et al., 2019). Although we did not detect a consistent increase in mIPSC amplitude [normalized amplitude $(\mathrm{NMDA} /$ control $)=1.13,95 \%$ confidence interval: $0.99-1.27, P=0.10$ ], we did detect a small enhancement in charge transfer [normalized charge $(\mathrm{NMDA} /$ control $)=$ 1.23, 95\% CI: $1.09-1.38, P=0.01$ ] accompanied by an increase in mIPSC $20 \%-80 \%$ decay (control $=7.74$ millisecond, $95 \%$ confidence interval: $5.91-9.56$ vs. $\mathrm{NMDA}=8.54$ millisecond, confidence interval: 7.10-9.98, $P=0.04$ ). In contrast, we found no associated change in mIPSC frequency [normalized frequency $(\mathrm{NMDA} / \mathrm{control})=0.98,95 \%$ confidence interval: $0.74-1.22, P=0.52]$. Thus, NMDA can cause elevations in intracellular calcium and enhance GlyR mIPSCs in the culture system if the internal solution's calcium buffering is low; however, it was not adequate to induce the substantial enhancements reported in Liu et al. (2010).

Species Difference Does Not Account for the Discrepancy. In this study, we recorded glycine currents in neurons derived from mouse spinal cord, whereas the original experiments were carried out in cultures derived from rat spinal cord (Liu et al., 2010). We hypothesized that species difference in GlyR composition/function might account for the lack of AP5 effect. Hence, we prepared parasagittal spinal cord slices from adult Sprague Dawley rats and recorded mIPSCs in the presence of TTX from lamina II neurons in the dorsal horn (Fig. 4C). AP5 did not alter the amplitude, charge, or frequency of glycine mIPSC recorded from rat spinal cord neurons [normalized amplitude $(\mathrm{AP} 5 /$ control $)=0.81,95 \%$ confidence interval: $0.57-1.1 ; P=0.07$; normalized charge (AP5/ control $)=0.84,95 \%$ confidence interval: $0.66-1.0 ; P=0.07$; normalized frequency (AP5/control) $=1.1,95 \%$ confidence interval $=0.55-1.5 ; P=0.80$, two-tailed paired $t$ test $]$.

Zinc Is a High-Affinity Positive Allosteric Modulator of GlyR and a Possible Contaminant. Finally, we hypothesized that a high-affinity PAM contaminant of GlyRs may have been responsible for the enhancement of GlyR mIPSCs reported by Liu et al. (2010). Zinc is a well characterized PAM of GlyRs (Bloomenthal et al., 1994; Lynch et al., 1998) and can potentiate GlyR currents by up to $\sim 200 \%$ at concentrations between $1 \mathrm{nM}$ and $5 \mu \mathrm{M}$ (Suwa et al., 2001; Miller et al., 2005). We tested the ability of $2 \mu \mathrm{M}$ free zinc $\left(200 \mu \mathrm{M} \mathrm{ZnCl} \mathrm{Zn}_{2}\right.$ in extracellular solution containing $10 \mathrm{mM}$ of the metal chelator, tricine) to enhance glycine mIPSCs (Fig. 5, A-D). Two micromolars free zinc strongly increased mIPSC amplitude and charge transfer without affecting frequency [normalized amplitude (zinc/control) $=1.38,95 \%$ confidence interval: $1.15-1.62, P=0.03$; normalized charge (zinc/control) $=2.00$, 95\% confidence interval: $1.15-1.62, P=0.001$; normalized frequency (zinc/control) $=0.88,95 \%$ confidence interval: $0.70-1.06, P=0.36$, two-tailed paired $t$ tests]. mIPSC 20\%$80 \%$ decay was increased by $62 \%$ (control $=6.60$ millisecond, 95\% confidence interval: $5.32-7.885$ vs. zinc $=10.7$ millisecond, confidence interval: 7.33-14.07, $P=0.01$, data not shown) As zinc contamination of biologic solutions can occur within seconds of their contact with a range of common laboratory materials (Kay, 2004) and can reach levels that are sufficient to enhance GlyR currents by 30\%-40\% (Suwa et al., 2001; Cornelison and Mihic, 2014), we tested the ability of very low concentrations of free zinc to alter glycine mIPSCs in our system. We found that $50 \mathrm{nM}$ free zinc $\left(5 \mu \mathrm{M} \mathrm{ZnCl}_{2}\right.$ in extracellular solution containing $10 \mathrm{mM}$ tricine) increased glycine mIPSC amplitudes by a maximum of $40 \%$ [normalized amplitude (zinc/control) $=1.19$, 95\% confidence interval: $1.04-1.34, P=0.02$ ], with consistent enhancement of charge transfer [normalized charge (zinc/control) $=1.66$, 95\% confidence interval: $1.42-1.90, P=0.001$ ] and no change in frequency [normalized frequency (zinc/control) $=$ 1.00, 95\% confidence interval: $0.74-1.25, P=0.001$, twotailed paired $t$ test]. This was accompanied by a $52 \%$ increase in mIPSC $20 \%-80 \%$ decay time (control $=7.76$ millisecond, $95 \%$ confidence interval: $4.95-10.56$ vs. zinc $=11.81$ millisecond, confidence interval: $9.50-14.14, P=0.002)$. Thus, solution contamination by a high-affinity glycine receptor PAM like zinc could have contributed to the results reported by Liu et al. (2010).

\section{Discussion}

We were unable to replicate the finding that AP5, NMDA, and glutamate are PAMs of GlyRs. We recorded GlyR-mediated 


\section{A 10mM BAPTA intracellular}
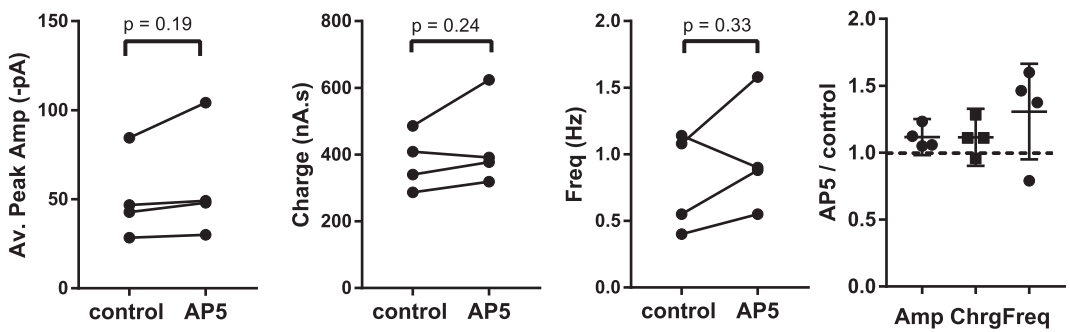

\section{B}
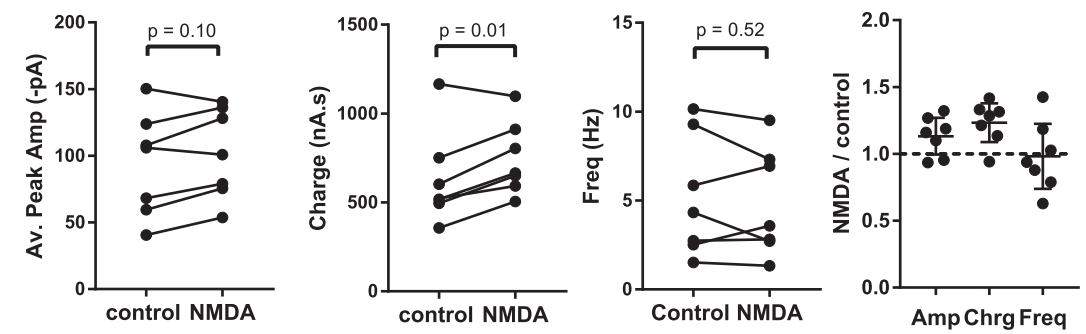

\section{Rat Spinal Cord Slice}
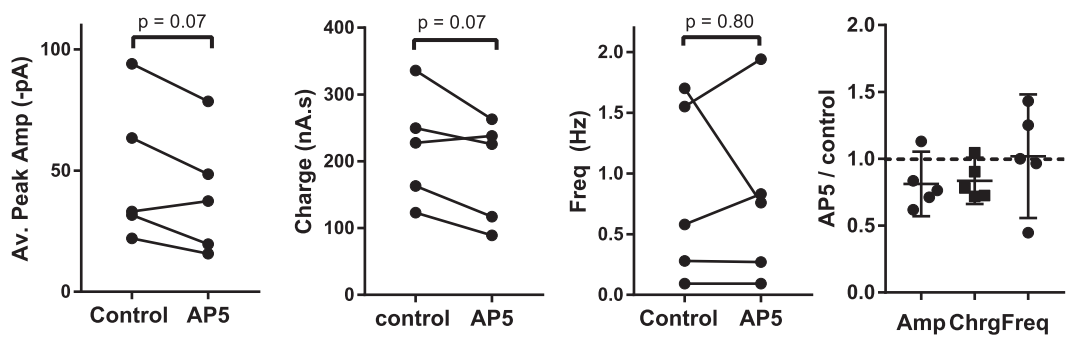

Fig. 4. The lack of AP5 effect is not due to intracellular calcium elevations or species difference. (A) We confirmed that AP5 did not alter the average peak amplitude, charge, and frequency of glycine mIPSCs recorded from mouse embryonic cultures if the intracellular recording solution contained $10 \mathrm{mM}$ BAPTA. (B) When the calcium buffering capacity of the intracellular recording solution was reduced (0.6 mM EGTA intracellular), mIPSCs were recorded in control conditions and after NMDA $(50 \mu \mathrm{M})$ was added to the bath solution. mIPSC peak amplitude and frequency remained stable; however, a small increase in mIPSC charge, reflecting a change in mIPSC kinetic, was observed. Normal extracellular solution containing calcium and magnesium was used for these experiments. (C) Next, we recorded miniature glycine IPSCs from neurons in lamina II of rat parasagittal spinal cord slices. Average mIPSC peak amplitude, charge, and frequency before (control) and after AP5 (100 $\mu \mathrm{M})$ exposure did not change. Data points from individual experiments are connected by a line, and in the right-hand panel, the proportional change (drug/control) of mIPSC amplitude, charge, and frequency is shown. currents in response to spontaneous and evoked synaptic release of glycine, as well as by exogenous bath-applied glycine. We tested the ability of AP5, NMDA, and glutamate to enhance glycine effects on recombinant human GlyRs at low and high glycine concentrations and in rat neurons but did not uncover any direct action of these compounds on GlyRs. Furthermore, only a subtle GlyR enhancement was detected when the calcium buffering capacity of our intracellular solution was reduced ( $0.6 \mathrm{mM}$ EGTA). Thus, we conclude that our lack of success in reproducing the results of Liu et al. (2010) cannot be explained by differences in subunit composition or species, nor by receptor saturation or indirect facilitation of GlyRs when intracellular calcium elevations occur. A recently published paper showing that glycine eIPSCs are stable in the presence of NMDA $(50 \mu \mathrm{M})$ plus the noncompetitive NMDAR antagonist 7-chlorokynurenic acid (Kloc et al., 2019) is consistent with our findings.

Finally, we confirmed that the well characterized GlyR PAM zinc strongly potentiated GlyR mIPSCs and showed that the potentiation could still be detected when zinc concentrations were as low as $50 \mathrm{nM}$. We propose that glutamate, AP5, and NMDA do not directly interact with GlyR to enhance currents and that contamination of the solutions used by Liu et al. (2010) by zinc or some other high-affinity PAM could conceivably be the origin of this error.
Methodological Differences. We were careful to replicate the key methodical conditions described in Liu et al. (2010). Neither the slight differences in internal pipette solution (Fig. 4) nor species or subunit differences were responsible for the lack of effect observed. Similar to the original study, we pre-exposed neurons to AP5/NMDA for $>3-5$ minutes before recording mIPSCs, eIPSCs, or exogenous glycine currents in neurons and recombinant human GlyRs expressed in oocytes for $>1$ minute before evoking glycine currents.

Cell Selection. We considered the possibility that the GlyR enhancement reported in Liu et al. (2010) was the result of the enrichment of a GlyR subtype that is uncommon in our cell cultures (which predominately express $\alpha 2$-containing GlyRs; Hoch et al., 1989) or the unconscious selection of different neuronal subsets by the two groups. However, AP5 did not alter GlyR mIPSCs recorded in adult rat spinal cord or oocytes expressing GlyR made from $\alpha 1, \alpha 3, \alpha 1 \beta$, or $\alpha 3 \beta$ subunits. Additionally, the original study also described the effect on GlyRs expressed in HEK cells. Together, these findings indicate that the cell/receptor population sampled is not likely to be a significant source of the difference between this study and the study of Liu et al. (2010).

Intracellular Calcium Elevations. Intracellular calcium changes signal a plethora of modifications to neuronal function. Many reports have shown that GlyR currents are 
A

\section{$2 \mu \mathrm{M}$ free zinc}

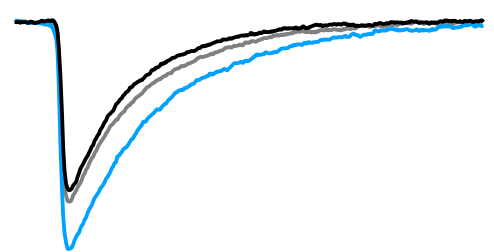

B

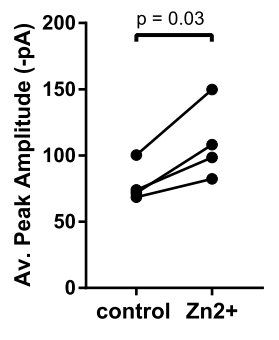

D
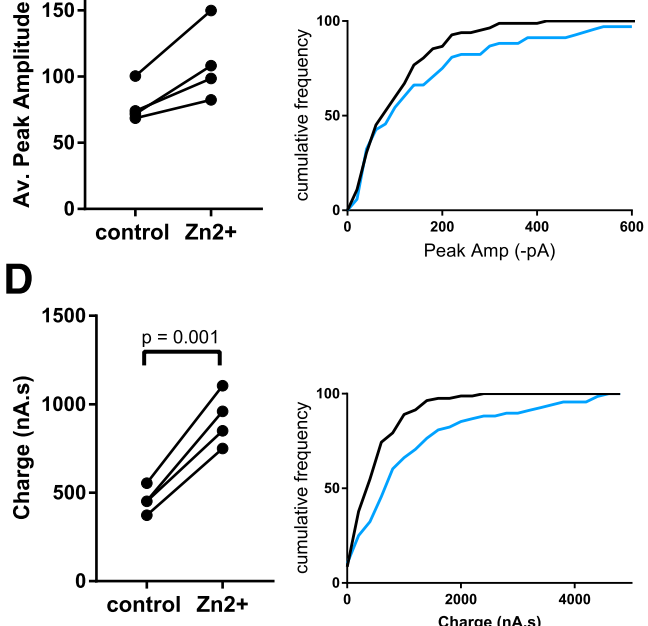

C
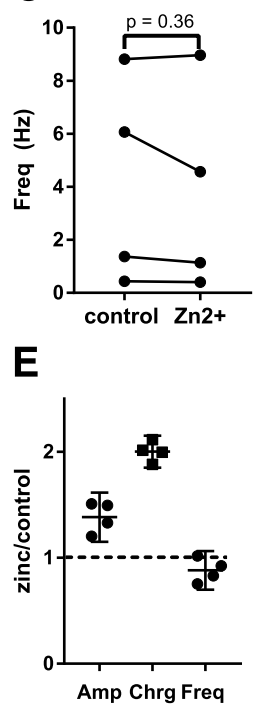

H

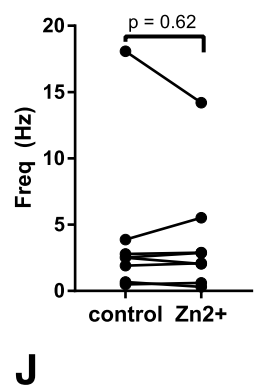

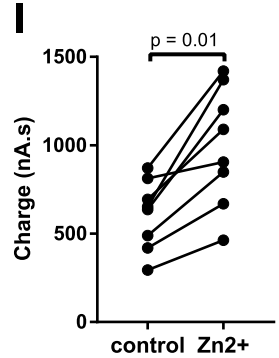

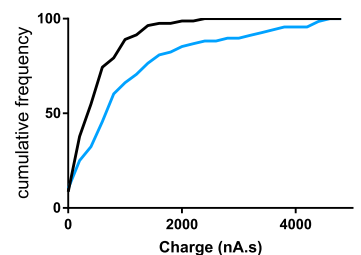

G
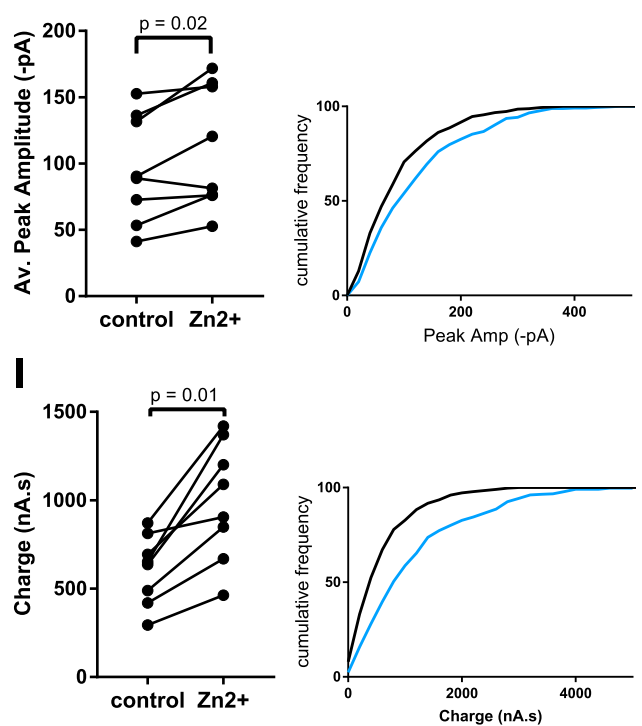

Fig. 5. Nanomolar concentrations of zinc enhance glycine mIPSCs. (A) An example of average glycine mIPSC traces before (control, black line) and after application of $\mathrm{Zn}^{2+}$ (blue line) and after washout (gray line) of zinc. Two micromolars free zinc (200 $\mu \mathrm{M}$ zinc in extracellular solution containing $10 \mathrm{mM}$ tricine) increased the average mIPSC peak amplitude (A and B) and charge transfer (A and D) but not the frequency (C). (E) The proportional change (zinc/control) of mIPSC amplitude, charge, and frequency in each condition. (F) An example of average glycine mIPSC traces before (control, black line) and after application of $\mathrm{Zn}^{2+}$ (blue line) and after washout (gray line) of zinc. The peak amplitude ( $\mathrm{F}$ and $\mathrm{G}$ ) and charge (F and I), but not the frequency (H) of mIPSCs was also increased when the free zinc concentration was reduced to $50 \mathrm{nM}(5 \mu \mathrm{M}$ zinc in extracellular solution containing $10 \mathrm{mM}$ tricine). (J) The proportional change (zinc/control) of mIPSC amplitude, charge, and frequency in each condition.

indirectly enhanced by stimulations that trigger elevations in intracellular calcium (Fucile et al., 2000; Xu et al., 2000; Lévi et al., 2008; Kloc et al., 2019) and GABA $\mathrm{As}_{\mathrm{s}}$ (Stelzer and Wong, 1989). Thus, any uncontrolled depolarization of the network (unlikely when TTX is present) or a contaminant that causes a receptor-mediated (e.g., NMDAR) elevation in intracellular calcium could, in theory, be the origin of the data reported in Liu et al. (2010). However, our data show that even when the intracellular solution used had low calcium buffering capacity (0.6 mM EGTA), mIPSCs were only slightly enhanced.

Contaminants. Like other ligand-gated ion channels, glycine receptors have multiple allosteric binding sites able to unlock the receptor and substantially increase glycine receptor-mediated signaling. In addition to zinc- and intracellular $\mathrm{Ca}^{2+}$-dependent processors, there are a host of other compounds that have been reported to enhance glycine receptor function. These include anesthetics (Mihic et al., 1997), cannabinoids and other fatty compounds (Hejazi et al., 2005; Yang et al., 2008), alcohol (Burgos et al., 2015; Lara et al., 2019), glucose (Breitinger et al., 2015), and others (Yevenes and Zeilhofer, 2011; Huang et al., 2016). One interesting compound is oleamide, a fatty acid amide that is present in the cerebrospinal fluid of sleep-deprived animals. Oleamide is also commonly used as a polymer lubricant and one of several 
bioactive contaminants known to leach from disposable laboratory plasticware (McDonald et al., 2008; Jug et al., 2020). Oleamide dose-dependently and stereospecifically potentiates $\mathrm{GABA}_{\mathrm{A}}$ and GlyRs (Coyne et al., 2002) and could have contributed to the GlyR effect reported by Liu et al. (2010).

As the GlyR enhancement observed by Liu et al. was reported to occur in response to many different compounds, detectable at the level of single-channel recordings, and present in recordings from recombinant GlyRs, we believe that it is likely that a known or unknown PAM contaminant in some key component of the extracellular solution is responsible for the GlyR enhancement observed by Liu et al. (2010). The presumptive contaminant could conceivably be part of the vehicle or storage container that the drug solutions were made in or might have leached from the tubing that delivered test compounds. Our data suggest that $50 \mathrm{nM}$ zinc, a concentration easily reached by solution contamination from labware, could have resulted in GlyR effects reported by Liu et al. (2010): it can enhance GlyR currents to the same level and is known to increase single-channel burst duration (Laube et al., 1995, 2000); however, Liu et al. (2010) did not report a change in mIPSC kinetics.

Glycine itself may be a contaminant of solutions, as it is a breakdown product of microbial contaminants. It is possible that drug solutions dissolved in water-based vehicle and used over extended periods may contain biologically active concentrations of glycine, especially if stored at room temperature (Hamilton and Myoda, 1974). Alternatively, contamination could arise from the drug application system. For example, if the control and test solutions were always delivered from the same drug reservoir, a low-level contamination with a highaffinity PAM could accumulate in the drug delivery system. Hydrophobic compounds like cannabinoids are particularly "sticky." In practice, there are multiple plausible sources for GlyR PAM contaminants in the laboratory.

Other possibilities considered were that sequentially applied compounds could interact with the GlyR to expose and bind to an unusual PAM binding site, or that the compounds used uncovered a strychnine sensitive current that does not originate from the glycine receptor. However, given Liu et al. (2010) were able to detect GlyR enhancement in recombinant systems and carried out single-channel recordings, these possibilities are unlikely.

Moving forward, the application of appropriate experimental protocols is important, and the appropriateness of a protocol should always be the first thing to be questioned when highly surprising and unexpected results are obtained. We suggest that any study characterizing a high-affinity PAM must include controls that eliminate the possibility of contamination to prevent misinterpretation of results.

Common sources of zinc contamination have been meticulously assessed (Kay, 2004; Cornelison and Mihic, 2014). Zinc (and other metal) contamination can be limited by making solutions fresh from water filtered with a high-grade purification system, always using high purity reagents, avoiding solution contact with glass, stainless steel or latex gloves, and avoiding polyethylene and polystyrene pipettes/tubing (Kay, 2004; Cornelison and Mihic, 2014). For drug targets like the GlyR, where zinc modulation occurs at concentrations of $<100 \mathrm{nM}$, a metal chelator such as EGTA, tricine, or diethylenetriaminepentaacetic acid (Paoletti et al., 1997; Kay, 2004) should be used.
In addition, controls addressing contaminants that may arise from leaching labware and during sample handling/preparation (see McDonald et al., 2008; Jug et al., 2020) should be considered, and it may be appropriate to include details about how drug stocks were prepared and the composition and manufacturer of plastic and glass laboratory equipment used (including coverslips, storage tubes, and drug delivery systems) in the methods section of the paper.

Contaminants including bioactive lipophilic compounds retained within the application system can be controlled for by designing the system with a minimal number of fittings, a minimal void volume, scrambling or randomization of the order of experiments, regular cleaning, and regular replacement of tubing. We use polyethylene tubing and wash our drug delivery system regularly with $70 \% \mathrm{v} / \mathrm{v}$ ethanol to prevent this sort of contamination.

Main Conclusions. This study disputes the previously published finding that GlyRs are directly modulated by AP5, NMDA, and glutamate and indicates that the findings of Liu et al. (2010) may have resulted from a misinterpretation of results due to a high-affinity GlyR PAM contaminant in the experimental system.

Therefore, GlyRs are not directly (and transiently) facilitated by glutamate spillover onto nearby inhibitory synapses, which would rely on factors such as synaptic glutamate levels, synaptic architecture, receptor expression patterns, and transporter performance (Huang, 1998; Turecek and Trussell, 2001; Ahmadi et al., 2003; Kubota et al., 2010). In contrast, the indirect glutamate-mediated, calcium-dependent enhancement of GlyRs will still occur when NMDARs are activated, after neuronal depolarization to relieve its block by magnesium (Fucile et al., 2000; Xu et al., 2000; Lévi et al., 2008; Kloc et al., 2019). This indirect modulation is mechanistically and temporally different from the direct PAM effects proposed by Liu et al. (2000) and would have distinct functional effects. Thus, this negative finding changes our understanding of how inhibitory and excitatory activity is balanced and regulated in the hindbrain.

The brain is designed to adapt and respond to subtle changes in input and is modulated by compounds that can have exceptionally high affinities (picomolars-micromolars) for their biologic targets. This is important because trace levels of unknown and unexpected contaminants can have profound physiologic effects. As a result, new high-affinity modulators must be presented with carefully designed controls to rule out common contaminates and detail how drugs were prepared, stored, and applied. Finally, the publication of replication studies by independent laboratories is essential to consolidating knowledge and more quickly advance understanding and progress in the field of neurobiology and drug development.

\section{Acknowledgments}

Thanks to Bryony Winters, Sherelle Casey, and Thomas Harmon for providing critical feedback and Prue and Andy Kennard for their continued interest and support.

\footnotetext{
Authorship Contributions

Participated in research design: Aubrey, Otsu.

Conducted experiments: Aubrey, Sheipouri.

Performed data analysis: Aubrey, Sheipouri.

Wrote or contributed to the writing of the manuscript: Aubrey, Sheipouri, Balle, Vandenberg, Otsu.
} 


\section{References}

Ahmadi S, Muth-Selbach U, Lauterbach A, Lipfert P, Neuhuber WL, and Zeilhofer HU (2003) Facilitation of spinal NMDA receptor currents by spillover of synaptically released glycine. Science 300:2094-2097.

Anderson WB, Graham BA, Beveridge NJ, Tooney PA, Brichta AM, and Callister RJ (2009) Different forms of glycine- and GABA(A)-receptor mediated inhibitory synaptic transmission in mouse superficial and deep dorsal horn neurons. Mol Pain 5:65.

Aubrey KR, Rossi FM, Ruivo R, Alboni S, Bellenchi GC, Le Goff A, Gasnier B, and Supplisson S (2007) The transporters GlyT2 and VIAAT cooperate to determine the vesicular glycinergic phenotype. J Neurosci 27:6273-6281.

Bloomenthal AB, Goldwater E, Pritchett DB, and Harrison NL (1994) Biphasic modulation of the strychnine-sensitive glycine receptor by $\mathrm{Zn} 2+$. Mol Pharmacol 46:1156-1159.

Breitinger U, Raafat KM, and Breitinger H-G (2015) Glucose is a positive modulator for the activation of human recombinant glycine receptors. J Neurochem 134 $1055-1066$

Burgos CF, Muñoz B, Guzman L, and Aguayo LG (2015) Ethanol effects on glycinergic transmission: From molecular pharmacology to behavior responses. Pharmacological Research 101:18-29.

Chaudhry FA, Reimer RJ, Bellocchio EE, Danbolt NC, Osen KK, Edwards RH, and Storm-Mathisen J (1998) The vesicular GABA transporter, VGAT, localizes to synaptic vesicles in sets of glycinergic as well as GABAergic neurons. $J$ Neurosci 18:9733-9750

Chéry N and de Koninck Y (1999) Junctional versus extrajunctional glycine and GABA(A) receptor-mediated IPSCs in identified lamina I neurons of the adult rat spinal cord. J Neurosci 19:7342-7355.

Chéry N and De Koninck Y (2000) GABA(B) receptors are the first target of released GABA at lamina I inhibitory synapses in the adult rat spinal cord. J Neurophysiol 84:1006-1011.

Colloca L, Ludman T, Bouhassira D, Baron R, Dickenson AH, Yarnitsky D, Freeman R, Truini A, Attal N, Finnerup NB, et al. (2017) Neuropathic pain. Nat Rev Dis Primers 3:17002

Cornelison GL and Mihic SJ (2014) Contaminating levels of zinc found in commonlyused labware and buffers affect glycine receptor currents. Brain Res Bull 100:1-5.

Coyne L, Lees G, Nicholson RA, Zheng J, and Neufield KD (2002) The sleep hormone oleamide modulates inhibitory ionotropic receptors in mammalian CNS in vitro. $\mathrm{Br}$ $J$ Pharmacol 135:1977-1987.

Foster E, Wildner H, Tudeau L, Haueter S, Ralvenius WT, Jegen M, Johannssen H, Hösli L, Haenraets K, Ghanem A, et al. (2015) Targeted ablation, silencing, and activation establish glycinergic dorsal horn neurons as key components of a spinal gate for pain and itch. Neuron 85:1289-1304.

Fucile S, De Saint Jan D, de Carvalho LP, and Bregestovski P (2000) Fast potentiation of glycine receptor channels of intracellular calcium in neurons and transfected cells. Neuron 28:571-583.

Hamilton PB and Myoda TT (1974) Contamination of distilled water, $\mathrm{HCl}$, and $\mathrm{NH} 4 \mathrm{OH}$ with amino acids, proteins, and bacteria. Clin Chem 20:687-691.

Hanus C (2004) Intracellular Association of Glycine Receptor with Gephyrin Increases Its Plasma Membrane Accumulation Rate. Journal of Neuroscience 24 $1119-1128$

Harvey RJ, Depner UB, Wässle H, Ahmadi S, Heindl C, Reinold H, Smart TG, Harvey K, Schütz B, Abo-Salem OM, et al. (2004) GlyR alpha3: an essential target for spinal PGE2-mediated inflammatory pain sensitization. Science 304:884-887.

Heinke B, Ruscheweyh R, Forsthuber L, Wunderbaldinger G, and Sandkühler J (2004) Physiological, neurochemical and morphological properties of a subgroup of GABAergic spinal lamina II neurones identified by expression of green fluorescent protein in mice. $J$ Physiol 560:249-266.

Hejazi N, Zhou C, Oz M, Sun H, Ye JH, and Zhang L (2005) $\Delta$ 9-Tetrahydrocannabinol and Endogenous Cannabinoid Anandamide Directly Potentiate the Function of Glycine Receptors. Mol Pharmacol 69:991-997.

Hoch W, Betz H, and Becker CM (1989) Primary cultures of mouse spinal cord express the neonatal isoform of the inhibitory glycine receptor. Neuron 3:339-348.

Huang EP (1998) Synaptic transmission: spillover at central synapses. Curr Biol 8 R613-R615.

Huang X, Shaffer PL, Ayube S, Bregman H, Chen H, Lehto SG, Luther JA, Matson DJ, McDonough SI, Michelsen K, et al. (2016) Crystal structures of human glycine receptor $\alpha 3$ bound to a novel class of analgesic potentiators. Nat Struct Mol Biol 24: 108-113.

Johnson JW and Ascher P (1987) Glycine potentiates the NMDA response in cultured mouse brain neurons. Nature 325:529-531.

Jonas P, Bischofberger J, and Sandkühler J (1998) Corelease of two fast neurotransmitters at a central synapse. Science 281:419-424.

Jug U, Naumoska K, Metlièar V, Schink A, Makuc D, Vovk I, Plavec J, and Lucas K (2020) Interference of oleamide with analytical and bioassay results. Sci Rep 10:2163.

Kay AR (2004) Detecting and minimizing zinc contamination in physiological solutions. BMC Physiol 4:4.

Keller AF, Coull JA, Chery N, Poisbeau P, and De Koninck Y (2001) Region-specific developmental specialization of GABA-glycine cosynapses in laminas I-II of the rat spinal dorsal horn. $J$ Neurosci 21:7871-7880.

Kloc ML, Pradier B, Chirila AM, and Kauer JA (2019) NMDA receptor activation induces long-term potentiation of glycine synapses. PLoS One 14:e0222066.

Kotak VC, Korada S, Schwartz IR, and Sanes DH (1998) A developmental shift from GABAergic to glycinergic transmission in the central auditory system. $J$ Neurosci 18:4646-4655.

Kubota H, Alle H, Betz H, and Geiger JR (2010) Presynaptic glycine receptors on hippocampal mossy fibers. Biochem Biophys Res Commun 393:587-591.

Kullmann DM (2000) Spillover and synaptic cross talk mediated by glutamate and GABA in the mammalian brain. Prog Brain Res 125:339-351.
Lara CO, Burgos CF, Silva-Grecchi T, Muñoz-Montesino C, Aguayo LG, Fuentealba J, Castro PA, Guzmán JL, Corringer P-J, Yévenes GE, et al. (2019) Large Intracellular Domain-Dependent Effects of Positive Allosteric Modulators on Glycine Receptors. ACS Chem Neurosci 10:2551-2559.

Laube B, Kuhse J, and Betz H (2000) Kinetic and mutational analysis of Zn2+ modulation of recombinant human inhibitory glycine receptors. $J$ Physiol 522:215-230.

Laube B, Kuhse J, Rundström N, Kirsch J, Schmieden V, and Betz H (1995) Modulation by zinc ions of native rat and recombinant human inhibitory glycine receptors. J Physiol 483:613-619.

Lévi S, Schweizer C, Bannai H, Pascual O, Charrier C, and Triller A (2008) Homeostatic regulation of synaptic GlyR numbers driven by lateral diffusion. Neuron 59:261-273.

Lim R, Alvarez FJ, and Walmsley B (2000) GABA mediates presynaptic inhibition at glycinergic synapses in a rat auditory brainstem nucleus. J Physiol 525:447-459.

Liu J, Wu DC, and Wang YT (2010) Allosteric potentiation of glycine receptor chloride currents by glutamate. Nat Neurosci 13:1225-1232.

$\mathrm{Lu}$ T, Rubio ME, and Trussell LO (2008) Glycinergic transmission shaped by the corelease of GABA in a mammalian auditory synapse. Neuron 57:524-535.

Lynch JW, Jacques P, Pierce KD, and Schofield PR (1998) Zinc potentiation of the glycine receptor chloride channel is mediated by allosteric pathways. $J$ Neurochem 71:2159-2168.

Marín O (2012) Interneuron dysfunction in psychiatric disorders. Nat Rev Neurosci 13:107-120.

McDonald GR, Hudson AL, Dunn SM, You H, Baker GB, Whittal RM, Martin JW, Jha A, Edmondson DE, and Holt A (2008) Bioactive contaminants leach from disposable laboratory plasticware. Science 322:917.

Mihic SJ, Ye Q, Wick MJ, Koltchine VV, Krasowski MD, Finn SE, Mascia MP Valenzuela CF, Hanson KK, Greenblatt EP, et al. (1997) Sites of alcohol and volatile anaesthetic action on GABA(A) and glycine receptors. Nature 389:385-389.

Miller PS, Da Silva HMA, and Smart TG (2005) Molecular basis for zinc potentiation at strychnine-sensitive glycine receptors. J Biol Chem 280:37877-37884

Mitchell EA, Gentet LJ, Dempster J, and Belelli D (2007) GABAA and glycine receptor-mediated transmission in rat lamina II neurones: relevance to the analgesic actions of neuroactive steroids. J Physiol 583:1021-1040.

Mitchell K, Spike RC, and Todd AJ (1993) An immunocytochemical study of glycine receptor and GABA in laminae I-III of rat spinal dorsal horn. J Neurosci 13:2371-2381.

Moore LA and Trussell LO (2017) Corelease of inhibitory neurotransmitters in the mouse auditory midbrain. J Neurosci 37:9453-9464.

Paoletti P, Ascher P, and Neyton J (1997) High-affinity zinc inhibition of NMDA NR1-NR2A receptors. J Neurosci 17:5711-5725.

Polgár E, Durrieux C, Hughes DI, and Todd AJ (2013) A quantitative study of inhibitory interneurons in laminae I-III of the mouse spinal dorsal horn. PLoS One 8:e78309

Rousseau F, Aubrey KR, and Supplisson S (2008) The glycine transporter GlyT2 controls the dynamics of synaptic vesicle refilling in inhibitory spinal cord neurons. $J$ Neurosci 28:9755-9768.

Sagné C, El Mestikawy S, Isambert MF, Hamon M, Henry JP, Giros B, and Gasnier B (1997) Cloning of a functional vesicular GABA and glycine transporter by screening of genome databases. FEBS Lett 417:177-183.

Stelzer A and Wong RKS (1989) GABAA responses in hippocampal neurons are potentiated by glutamate. Nature 337:170-173.

Sun H, Lu L, Zuo Y, Wang Y, Jiao Y, Zeng WZ, Huang C, Zhu MX, Zamponi GW, Zhou $\mathrm{T}$, et al. (2014) Kainate receptor activation induces glycine receptor endocytosis through PKC deSUMOylation. Nat Commun 5:4980.

Suwa H, Saint-Amant L, Triller A, Drapeau P, and Legendre P (2001) High-affinity zinc potentiation of inhibitory postsynaptic glycinergic currents in the zebrafish hindbrain. J Neurophysiol 85:912-925.

Todd AJ and Sullivan AC (1990) Light microscope study of the coexistence of GABAlike and glycine-like immunoreactivities in the spinal cord of the rat. J Comp Neurol 296:496-505.

Triller A and Choquet D (2005) Surface trafficking of receptors between synaptic and extrasynaptic membranes: and yet they do move!. Trends Neurosci 28:133-139.

Turecek R and Trussell LO (2001) Presynaptic glycine receptors enhance transmitter release at a mammalian central synapse. Nature 411:587-590.

Vaaga CE, Borisovska M, and Westbrook GL (2014) Dual-transmitter neurons: functional implications of co-release and co-transmission. Curr Opin Neurobiol 29:25-32.

Xu T-L, Dong X-P, and Wang D-S (2000) N-methyl-D-aspartate enhancement of the glycine response in the rat sacral dorsal commissural neurons. Eur $J$ Neurosci 12:1647-1653.

Yamanaka I, Miki M, Asakawa K, Kawakami K, Oda Y, and Hirata H (2013) Glycinergic transmission and postsynaptic activation of CaMKII are required for glycine receptor clustering in vivo. Genes Cells 18:211-224.

Yang Z, Aubrey KR, Alroy I, Harvey RJ, Vandenberg RJ, and Lynch JW (2008) Subunit-specific modulation of glycine receptors by cannabinoids and N-arachidonyl-glycine. Biochemical Pharmacology 76:1014-1023.

Yevenes GE and Zeilhofer HU (2011) Allosteric modulation of glycine receptors. $\mathrm{Br}$ $J$ Pharmacol 164:224-236.

Zeilhofer HU, Wildner H, and Yévenes GE (2012) Fast synaptic inhibition in spinal sensory processing and pain control. Physiol Rev 92:193-235.

Address correspondence to: Dr. Karin R. Aubrey, Pain Management Research Institute, Kolling Institute of Medical Research (Level 13), Royal North Shore Hospital and Faculty of Medicine and Health - Northern, University of Sydney, St Leonards, NSW 2065, Australia. E-mail: karin.aubrey@sydney.edu.au 